\section{AB0633 MEASURING WHAT MATTERS TO LUPUS PATIENTS: TRANSLATING PATIENT VIEWS INTO NOVEL PATIENT- REPORTED OUTCOMES}

S. Cleanthous ${ }^{1}$, A. Regnault ${ }^{2}$, M. Schneider ${ }^{3}$, C. Gordon ${ }^{4}$, S. Bartlett ${ }^{5}$, S. Cano ${ }^{1}$, T. Morel ${ }^{6} .{ }^{1}$ Modus Outcomes, Cambridge, UK; ${ }^{2}$ Modus Outcomes, Lyon, France; ${ }^{3}$ Policlinic and Hiller Research Unit of Rheumatology, UKD, Heinrich-HeineUniversity, Düsseldorf, Germany; ${ }^{4}$ University of Birmingham, Birmingham, UK ${ }^{5}$ McGill University, Montreal, Canada; ${ }^{6}$ UCB Pharma, Brussels, Belgium

Background: The 2010 U.S. FDA guidance ${ }^{1}$ on systemic lupus erythematosus (SLE) acknowledged that 'improvements in clinical outcome measures in patients with SLE may not always translate to improvements in how patients feel or function' and encourages the use of patient-reported outcome instruments (PROs) to assess changes in outcomes that matter most to patients. Fatigue, identified by patients as their chief complaint during the Lupus Patient-Focused Drug Development (PFDD) meeting ${ }^{2}$, is not optimally measured by existing PROs ${ }^{1}$. There is a need for new patient-centred outcome measures as they are pivotal to PFDD. To address this, UCB has been conducting a multi-faceted, patient-centric, mixed methods research programme including evidence generated from >2100 SLE patients.

Objectives: There were three main objectives of the programme: 1) identify what concepts are most important to measure for SLE patients when evaluating treatment benefit; 2) critically evaluate the extent to which these concepts are captured by existing PROs; and 3) explore the opportunity to develop and evaluate new conceptually-based PROs in SLE.

Methods: There were three stages: 1 ) development of a preliminary treatment benefit conceptual model related to SLE including disease symptoms and impacts (generated from literature review, and patient and clinician input); 2) formal comparison of i) the conceptual model and the content validity (qualitative review) of existing SLE PROs and ii) psychometric properties (ie. SF-36, FACIT-F, and LupusQoL in the discontinued EMBODY Phase 3 studies for epratuzumab); 3) development of new conceptually-based PROs in SLE (figure 1).

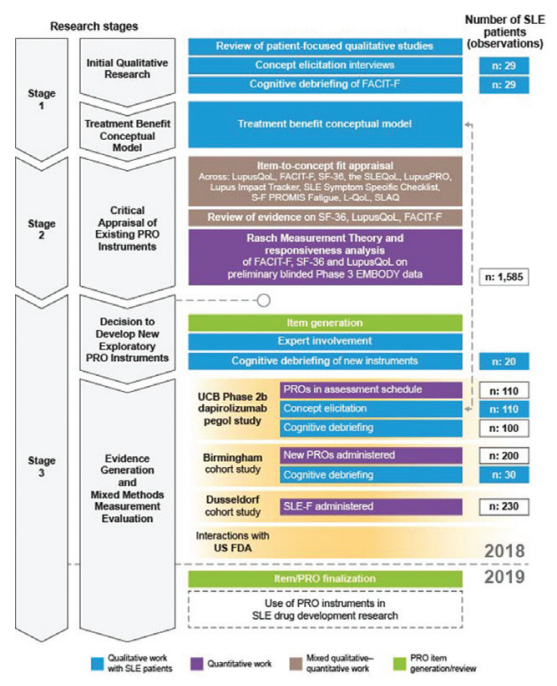

Abstract AB0633 - Figure 1

Results: A preliminary treatment benefit conceptual model in SLE was developed. Among the many symptoms and disease impacts reported by SLE patients, physical fatigue, mental fatigue ('brain fog'), sudden fatigability, joint/muscle stiffness and pain, skin symptoms and mobility difficulties featured as most troublesome. The content validity of 10 widely used PROs was found to be limited when compared to this conceptual model. Psychometric analysis (based on Rasch Measurement Theory) confirmed the limitations of the SLE PROs used in the EMBODY studies and suggested post-hoc reconceptualisation would improve the ability of the scales to detect clinical change. This led to the development of five new conceptually-based SLE PROs (ie. fatigue, pain, mobility, symptom severity, and emotional state), which are currently being explored in $>500$ SLE patients in a Phase $2 b$ dapirolizumab pegol study (NCT02804763) and two cross-sectional, non-interventional, observational studies. Data on the new PROs are currently being assessed at several sites in the USA, Latin America, UK and Germany and will be available in the coming months.

Conclusions: New, well-defined and reliable PROs to better capture the patient perceptions of the symptoms and impact of SLE are needed. UCB has developed five new PROs which offer the promise of improved patient-centred outcome measurements in SLE and other autoimmune diseases.

\section{REFERENCES:}

[1] Food and Drug Administration. Guidance for Industry. Systemic Lupus Erythematosus. June 2010

[2] Lupus Patient-Focused Drug Development meeting, 25 September 2017

Disclosure of Interest: S. Cleanthous Employee of: Modus Outcomes Ltd, A. Regnault Employee of: Modus Outcomes Ltd, M. Schneider Consultant for: UCB Pharma, C. Gordon Grant/research support from: UCB Pharma, Consultant for: UCB, Merck Serono, GSK, S. Bartlett: None declared, S. Cano Shareholder of: Modus Outcomes Ltd, Employee of: Modus Outcomes Ltd, T. Morel Shareholder of: UCB Pharma, Employee of: UCB Pharma

DOI: 10.1136/annrheumdis-2018-eular.5435

\section{AB0634 CHARACTERISATION OF SLE PATIENTS WHO UNDERWENT CARDIOVASCULAR SURGERY}

T. Kawamoto ${ }^{1}$, S. Matsushita ${ }^{2}$, H. Amano ${ }^{1}$, K. Minowa ${ }^{1}$, Y. Asai ${ }^{1}$, K. Yamaji ${ }^{1}$, A. Amano ${ }^{2}, \mathrm{~N}$. Tamura ${ }^{1} .{ }^{1}$ Internal medicine and rheumatlogy; ${ }^{2}$ Cardiovascular Surgery, Juntendo University School of Medicine, Tokyo, Japan

Background: SLE is characterised by alternate occurrences of flares and remissions throughout its course. During these flares, immune complexes are excessively produced and deposited in affected tissues. Previous reports suggest that immune complexes deposited in the blood vessels, atrioventricular valves, and other associated components of the heart cause disorders in these tissues. Although, the characteristics of patients in whom tissue injuries progress and ultimately require surgery have not been clarified. ${ }^{1}$

Objectives: Heart diseases are categorised into valvular disease, ischaemic heart disease, and aortic aneurysm. This study was conducted to determine whether there was a correlation between the activity of SLE and the progress and aggravation of heart disease in each category.

Methods: Of the 2707 patients who were diagnosed with SLE at Juntendo University Hospital from 2012-2017, 35 underwent cardiac surgery. Six patients met the exclusion criteria, and thus, 29 patients were included. Patients with infectious endocarditis and those in whom SLE had not been diagnosed prior to surgery were excluded. Indices for SLE activity were anti-DNA Ab and CH50. Peak and bottom values of these indices before surgery were recorded, and the values obtained during the pre-surgery 180 day period were compared with those that had been determined prior to this period.

Results: The mean disease duration at the time of cardiac surgery was 24.2 years. The cardiac diseases were heart valve disease $(\mathrm{V})$ in 10 , ischaemic heart disease (I) in 7, and aortic aneurysm (A) in 12 patients. The integrated values obtained by the activity indices, anti-DNA Ab, and disease duration were 14020 for V, 32966 for I, and 29444 for A. Thus, there was a tendency for values to be slightly lower in the heart valve disease category. The peak/bottom values for $\mathrm{CH} 50$ and anti-DNA Ab in the pre-surgery 180 day period were no different from those obtained prior to this period. Investigation of anti-phospholipid antibodies indicated that the rates of positive result for either $\mathrm{CL} \beta_{2} \mathrm{GPI}$, anti-CL antibodies, or LA were $60 \%$ for $V, 28 \%$ for $I$, and $33 \%$ for $A$, and thus, higher in the heart valve disease category. All patients were treated with steroids, and five patients $(17.2 \%)$ of the 29 used immunosuppressants. This rate tended to be lower than the previous frequency of the combined use of immunosuppressive drugs at our hospital (onset: $18.4 \%$, first flare $33.3 \%$ ). ${ }^{2}$

Conclusions: There is a possibility that the long-term duration of SLE may lead to cardiac disease requiring surgery. The integrated value for DNA Ab was lower in the heart valve disease category; therefore, the presence of anti-phospholipid antibodies may be a risk factor for valvular disease. The low frequency of combined use of immunosuppressants in SLE patients with cardiovascular manifestations requiring surgery, suggest the possibility that treatment with a single steroid may require heart surgery.

\section{REFERENCES:}

[1] Yasuhiro Sakaguti, Sachio Kawai, Ryozo Okada, et al. Immunohistochemical study of the endomyocardial biopsy of Systemic Lupus Erythematosus. Journal of Cardiology. 1995;25:181-188

[2] Kentaro Minowa, Hirofumi Amano, Yoshinari Takasaki, et al. Disease flare patterns and predictors of systemic lupus erythematosus in a monocentric cohort of 423 Japanese patients during long-term follow-up: The JUDE study. Mod Rheumatol. 2017;27:72-76

Acknowledgements: none

Disclosure of Interest: None declared

DOI: 10.1136/annrheumdis-2018-eular.5041 